\title{
A long-term strength of constructive materials
}

\author{
Evgeny Larionov ${ }^{1}$
}

Moscow State University of Civil Engineering, Yaroslavskoe shosse, 26, Moscow, 129337, Russia

\begin{abstract}
A long-term strength materials under an axially loading of constructive elements is considered and the estimates of this strength are reduced. The proposed approach is connected with the notion so-called energy of entirety [1]. It is notable that this value can be used instead of known Reiner's invariant [2]. A material (concrete, steel, graph) is considered as a union of its links with statistical disturbed strengths [3]. This conception allows to modify Boltzmann's principle superposition of fraction creep deformations [4] and in addition, implies the identity of non-linear stresses function for the instantaneous and retarding deformations. The degeneration of long-term strength because of vibrational influence take into account and the strengthening of the materials in the course of their formation is considered.
\end{abstract}

The problems of forecast of a long-term structural safety of buildings necessary are connected with the strength $R(\tau)$ of constructive materials, presenting their basic mechanical characteristic. Here $\tau$ is a current temps. A material is a thermodynamic system and in consequence of energetic and mass exchange with environment the generating $R(\tau)$ constraint forces are formed. The accumulated in materials specific energy $W(\tau)$ is called their energy of entirety [1]. These values define the energetic state of the materials and represent their maximal measure of resistance to a destruction [5]. Since the destruction at moment $\tau=t$ is generated by instantaneous deformations the corresponding specific work $A(t)$ is considered as the energetic parameter $W(t)$ of material, named the energy of its entirety. Beside of the force action the magnitude of energy $W(t)$ essentially depends from the corrosion damages [6]. If the destruction of material under constant over of period $\left[t_{0} \leq \tau \leq t\right]$ normal stress $R\left(t, t_{0}\right)$ take place at moment $\tau=t$, the value $R\left(t, t_{0}\right)$ presents its strength for this period of temps. Therefore the value $R\left(\infty, t_{0}\right)$ is called the long-term strength of this material.

The destruction of a part of the links under an increasing on cross-sections $G$ axially loading $N(\tau)$ results in the redistribution of $N(\tau)$ on cross-sections $G(\tau)$ of the capable to resist links. This generates a non-linear dependence of the deformations on the calculated stresses $\sigma(\tau)=N(\tau) / F$, obtained under the assumption of an equal strength for all links. In fact there is a linear dependence of the deformations of the undamaged links on

${ }^{1}$ Email: LarionovEA@mgsu.ru 
the so-called structural stresses $\sigma_{s}(\tau)=N(\tau) / F(\tau)$. Here $F$ and $F(\tau)$ are the area of $\mathrm{G}$ and $\mathrm{G}(\tau)$ respectively. Then we obtain the following correlation

$$
\sigma_{s}(\tau)=S(t) \sigma(\tau) ; S(t)=F / F(\tau),
$$

where $S(t)$ is called the function of non-linearity of stresses. The constant over of period $\left[t_{0}, t\right]$ stress $\sigma_{s}(\tau)$ generate the deformation

$$
\varepsilon\left(t, t_{0}\right)=\varepsilon_{i n s t}(t)+\varepsilon_{c r}\left(t, t_{0}\right)
$$

where $\varepsilon_{\text {inst }}(t)=\sigma_{s}(t) / E(t)$ is the instantaneous and $\varepsilon_{c r}\left(t, t_{0}\right)=C\left(t, t_{0}\right) \sigma_{s}(t)$ is the creep,deformation; is the measure of simple creep and is the elasticity module.

An increment $\Delta \sigma_{s}(t)=\sigma_{s}(t)-\sigma_{s}\left(t_{0}\right)$ implies the creep deformation $\Delta \varepsilon_{c r}\left(t, t_{0}\right)$. Since a fraction increment $\Delta \varepsilon_{c r}\left(t, \tau_{i}\right)=C\left(t, \tau_{i}\right) \Delta \sigma_{s}\left(\tau_{i}\right)$ is independent from a value and duration of the rest increments $\Delta \sigma_{s}\left(\tau_{j}\right) ; j \neq i$, we can define $\Delta \varepsilon_{c r}\left(t, t_{0}\right)$ by Boltzmann's principle of superposition. In result with respect to the structural stresses we reduce the linear theological state equation [7], [8].

$$
\varepsilon\left(t, t_{0}\right)=\left[\frac{1}{E(t)}+C(t, t)\right] \sigma_{s}(t)-\int_{t_{0}}^{t} \sigma_{s}(\tau) \frac{\partial C(t, \tau)}{\partial \tau} d \tau
$$

Here $C(t, t)$ is so-called quick creep [9].

The value $W^{f}(\tau)=W\left(t_{0}\right)-A(\tau)$ we call a current energetic reserve of entirety. The condition $W^{f}\left(\tau_{*}\right)=0$ means the exhaustion of this reserve, implying the destruction at moment $\tau=\tau_{*}$. In case $\tau_{*}=t_{0}$ we have the shirt-term destruction, when $\tau_{*}=t>t_{0}$ the destruction in end of $\left[t_{0}, t\right]$. Consider the exhaustion of $\mathscr{W}(\tau)$ only by force deformations and suppose that $E(\tau)=E\left(t_{0}\right) ; C(t, \tau)=C\left(t_{0}, t_{0}\right)$. This means that $W(\tau)=$ const ; $t_{0} \leq \tau \leq t$. Then for the performed by $R_{s}\left(t_{0}\right)$ and $R_{s}\left(t, t_{0}\right)$ specific works we obtain respectively

$$
\begin{gathered}
A\left(t_{0}\right)=R_{s}^{2}\left(t_{0}\right)\left[1+C\left(t_{0}, t_{0}\right) E\left(t_{0}\right)\right] / E\left(t_{0}\right), \\
A\left(t, t_{0}\right)=R_{s}^{2}\left(t, t_{0}\right)\left[1+C\left(t, t_{0}\right) E\left(t_{0}\right)\right] / E\left(t_{0}\right)
\end{gathered}
$$

Since from $W(\tau)=$ const it follows $A\left(t, t_{0}\right)=A\left(t_{0}\right)$ we have

$$
\begin{gathered}
R_{s}\left(t, t_{0}\right)=r\left(t, t_{0}\right) R_{s}\left(t_{0}\right), \\
r\left(t, t_{0}\right)=\sqrt{\frac{1+E\left(t_{0}\right) C\left(t_{0}, t_{0}\right)}{1+E\left(t_{0}\right) C\left(t, t_{0}\right)}} .
\end{gathered}
$$

In applications the function 


$$
S(\tau)=1+V\left[\frac{\sigma(\tau)}{R(\tau)}\right]^{m}
$$

is used, where $V, m$ are the empirical parameters [10]. Usually for the concrete is supposed $m=4$ and $C(t, \tau)=C_{\infty}\left[1-\beta e^{-\gamma(t-\tau)}\right] ; \beta=0,8$. Here $C_{\infty}$ is the measure of simple creep as $t \rightarrow \infty, \quad \gamma$ is the parameter of open surface. Thus we have $r\left(\infty, t_{0}\right)=\sqrt{\left[1+0,2 E\left(t_{0}\right) C_{\infty}\right] / 1+E\left(t_{0}\right) C_{\infty}}$. By relations (6) and (8) we obtain the equation

$$
V\left[R\left(\infty, t_{0}\right)\right]^{-5}+\left[R\left(t_{0}\right)\right]^{4} R\left(\infty, t_{0}\right)-(1+V) r\left(\infty, t_{0}\right)\left[R\left(t_{0}\right)\right]^{-5}=0
$$

with respect to $R\left(\infty, t_{0}\right)$. The positive root of this equation represents the desired estimate of $R\left(\infty, t_{0}\right)$.

In case of linear statement $S(\tau)=1$ and by $(6)$

$$
R\left(\infty, t_{0}\right)=r\left(\infty, t_{0}\right) R\left(t_{0}\right)
$$

It is known that the creep of statically loaded material (for example concrete) increase under vibrational influence. This phenvmenon is called the vibrocreep of material concrete. The following correlation

$$
C_{V}\left(t, t_{0}\right)=K_{V}(\omega, \gamma, \rho) C\left(t_{0}, t_{0}\right)
$$

was established on basis of experiments in [11], [12]. The factor $K_{V}(\omega, \gamma, \rho)$ is determined by frequency $\omega$, parameter $\gamma$ and asymmetry $\rho=\frac{\sigma_{\min }}{\sigma_{\max }}$ of stresses. This factor is studied by Davidenkov's invariant - the independence from $\omega$ of the dissiped in course of one cycle energy $\Delta W$ [13], [14]. Note that to this end the proposed in [1] deformative invariant also can be used. By the relations (6) and (11), taking into account the vibrocreep of materials, we have

$$
\begin{gathered}
R_{s}\left(t, t_{0}\right)=r_{V}\left(t, t_{0}\right) R_{s}\left(t_{0}\right) \\
r_{V}\left(t, t_{0}\right)=\sqrt{\left[1+E\left(t_{0}\right) C_{V}\left(t_{0}, t_{0}\right)\right] / 1+E\left(t_{0}\right) C_{V}\left(t_{0}, t_{0}\right)}
\end{gathered}
$$

In the course of formation the energy of entirety $W(\tau)$ of materials increases that results in the increase of the strength $R(\tau)$. Along with $R(\tau)$ the module $E(\tau)$ also increases. Following [15] the equality their functions of the strengthening is supposed

$$
\psi_{R}(\tau)=\psi_{E}(\tau)
$$

The process of strengthening is accompanied decreasing of $C(\tau, \tau)$. On basis of the experiments [11], [16] the following relations are reduced [1].

$$
R(\tau) C(t, \tau)=\text { const }
$$




$$
E(\tau) C(\tau, \tau)=\text { const }
$$

The independence of $I(\tau)=E(\tau) C(\tau, \tau)$ from $\tau$ means the expected compensation of the largement of $E(\tau)$ by the reduction of $C(\tau, \tau)$. By solution of described of variation of $E(\tau)$ differential equation we obtain

$$
\psi_{E}\left(t, t_{0}\right)=1+\left[\frac{E(t)}{E\left(t_{0}\right)}-1\right]\left[1-e^{-\lambda\left(t-t_{0}\right)}\right]
$$

Here $\lambda$ is a physico-chemical parameter of the strengthening. Then according to (6), (14) and (17) we have

$$
\begin{gathered}
R_{s}\left(t, t_{0}\right)=\gamma\left(t, t_{0}\right) R_{s}\left(t_{0}\right) \\
\gamma\left(t, t_{0}\right)=r\left(t, t_{0}\right)\left\{1+\left[\frac{R(t)}{R\left(t_{0}\right)}-1\right]\left[1-e^{-\lambda\left(t-t_{0}\right)}\right]\right\}
\end{gathered}
$$

The factor $\gamma\left(t, t_{0}\right)$ defines the current reserve of strength. Usually for concrete is supposed that $t_{0}=28$ day.

Note that the relation (6) at $R(\tau)=$ const follows from the equality (18).

Cite the concluding remarks.

1. In the above examined problem the application of $W(\tau)$ allows to obtain the estimates of $R\left(\infty, t_{0}\right)$ more simply than by previous approaches.

2. The accounting of strength reserve $\gamma\left(t, t_{0}\right)$ is of interest during of construction of the contemporary buildings.

\section{References}

1 E.A. Larionov, Long-term force resistance and safety of buildings. Dis. doct. sci., Moscow (2005)

2 M. Reiner, Rheology (Nauka 1965)

3 W. Weibull, Trans Roy Inst. Technol. (Stockholm), 27 (1949)

4 L. Boltzmann [WA-1,616] Wien, Ber. 70. S.275-306. (1874) und Pogg Ann. Erg.-Bd.7. S. 624 (1876)

5 E.A. Larionov, Math. Universities, Builbing, 8 (2005)

6 V. F. Stepanova, V.R. Falikman, Proc. of II Int. conf. on concr and reinfor concr. 7. 324-35 (2014)

7 V.M. Bondarenko, E.A. Larionov, Str. Mech. of. Eng. Constr. and Build. 2 (2011)

8 E.A. Larionov, A.E. Larionov, Str. Mech. and Calculi. build, 2 (2015)

9 Yu. N. Rabotnov, A creep of constructive elements (Nauka 1982)

10 K.Z. Galustov, Non-linear creep theory of concrete and calculation of reinforced constructions (Fizmatlit, 2006)

11 V.M. Bondarenko, S.V. Bondarenko, Engineering methods of non-linear theory of reinforced concrete (Stroyizdat, 1982) 
12 K.K. Shkerbelis, On connection between the creep deformations and the velocity of the loading (Riga, 1959)

13 N.N. Davidenkov, JEP, 4.VIII, 6 (1938)

14 V.M. Bondarenko, E.A. Larionov, Math. universities Building 3 (2004)

15 G.A. Geniev, Proc. OGTU, №2/14(530) (2007)

16 S.V. Alexsandrovskiy, V.V. Solomonov, Trans, of NIISP, M., 6 (1972) 